

\section{Study of charge-carrier relaxation in a disordered organic semiconductor by simulating impedance spectroscopy}

\section{Citation for published version (APA):}

Mesta, M., Cottaar, J., Coehoorn, R., \& Bobbert, P. A. (2014). Study of charge-carrier relaxation in a disordered organic semiconductor by simulating impedance spectroscopy. Applied Physics Letters, 104, 1-4. [213301]. https://doi.org/10.1063/1.4880355

DOI:

$10.1063 / 1.4880355$

Document status and date:

Published: 01/01/2014

\section{Document Version:}

Publisher's PDF, also known as Version of Record (includes final page, issue and volume numbers)

\section{Please check the document version of this publication:}

- A submitted manuscript is the version of the article upon submission and before peer-review. There can be important differences between the submitted version and the official published version of record. People interested in the research are advised to contact the author for the final version of the publication, or visit the $\mathrm{DOI}$ to the publisher's website.

- The final author version and the galley proof are versions of the publication after peer review.

- The final published version features the final layout of the paper including the volume, issue and page numbers.

Link to publication

\section{General rights}

Copyright and moral rights for the publications made accessible in the public portal are retained by the authors and/or other copyright owners and it is a condition of accessing publications that users recognise and abide by the legal requirements associated with these rights.

- Users may download and print one copy of any publication from the public portal for the purpose of private study or research.

- You may not further distribute the material or use it for any profit-making activity or commercial gain

- You may freely distribute the URL identifying the publication in the public portal.

If the publication is distributed under the terms of Article 25fa of the Dutch Copyright Act, indicated by the "Taverne" license above, please follow below link for the End User Agreement:

www.tue.nl/taverne

Take down policy

If you believe that this document breaches copyright please contact us at:

openaccess@tue.nl

providing details and we will investigate your claim. 


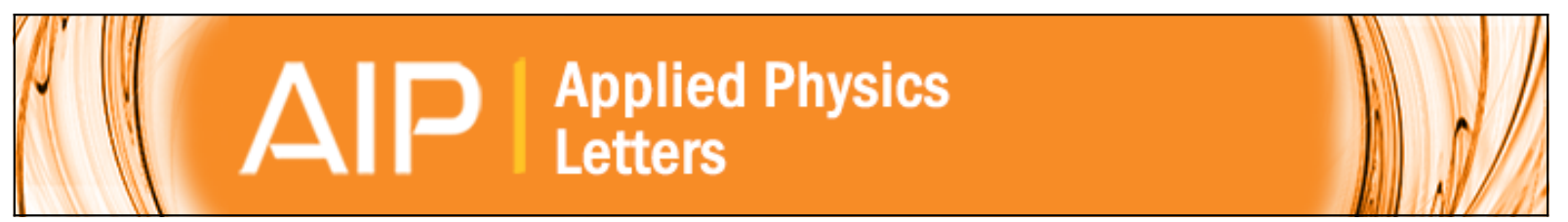

\section{Study of charge-carrier relaxation in a disordered organic semiconductor by simulating} impedance spectroscopy

M. Mesta, J. Cottaar, R. Coehoorn, and P. A. Bobbert

Citation: Applied Physics Letters 104, 213301 (2014); doi: 10.1063/1.4880355

View online: http://dx.doi.org/10.1063/1.4880355

View Table of Contents: http://scitation.aip.org/content/aip/journal/apl/104/21?ver=pdfcov

Published by the AIP Publishing

\section{Articles you may be interested in}

Charge carrier relaxation model in disordered organic semiconductors

AlP Advances 3, 112119 (2013); 10.1063/1.4834135

Analysis of interface carrier accumulation and relaxation in pentacene/C60 double-layer organic solar cell by impedance spectroscopy and electric-field-induced optical second harmonic generation

J. Appl. Phys. 110, 074509 (2011); 10.1063/1.3642964

Modeling carrier transport and electric field evolution in Gaussian disordered organic field-effect transistors J. Appl. Phys. 109, 104512 (2011); 10.1063/1.3590154

Transport limited interfacial carrier relaxation in a double-layer device investigated by time-resolved second harmonic generation and impedance spectroscopy

Appl. Phys. Lett. 98, 092109 (2011); 10.1063/1.3560054

Charge carrier dynamics in organic semiconductors by position dependent optical probing J. Appl. Phys. 101, 064510 (2007); 10.1063/1.2711390

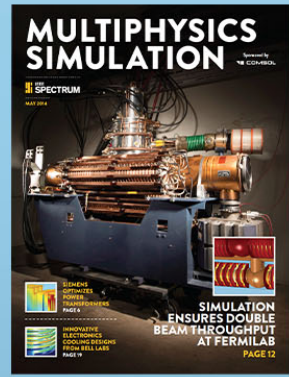

Free online magazine






\title{
Study of charge-carrier relaxation in a disordered organic semiconductor by simulating impedance spectroscopy
}

\author{
M. Mesta, ${ }^{1}$ J. Cottaar, ${ }^{1}$ R. Coehoorn, ${ }^{2,1}$ and P. A. Bobbert ${ }^{1}$ \\ ${ }^{1}$ Department of Applied Physics, Technische Universiteit Eindhoven, P.O. Box 513, NL-5600 MB Eindhoven, \\ The Netherlands \\ ${ }^{2}$ Philips Research Laboratories, High Tech Campus 4, NL-5656 AE Eindhoven, The Netherlands
}

(Received 27 March 2014; accepted 9 May 2014; published online 27 May 2014)

\begin{abstract}
Impedance spectroscopy is a very sensitive probe of nonstationary charge transport governed by charge-carrier relaxation in devices of disordered organic semiconductors. We simulate impedance spectroscopy measurements of hole-only devices of a polyfluorene-based disordered organic semiconductor by solving a time-dependent three-dimensional master equation for the occupational probabilities of transport sites in the semiconductor. We focus on the capacitance-voltage characteristics at different frequencies. In order to obtain good agreement with the measured characteristics, we have to assume a lower strength of a Gaussian energy disorder than obtained from best fits to the stationary current density-voltage characteristics. This lower disorder strength is in agreement with dark-injection studies of nonstationary charge transport on the same devices. The results add to solving the puzzle of reconciling nonstationary with stationary charge-transport studies of disordered organic semiconductors. @ 2014 AIP Publishing LLC.
\end{abstract}

[http://dx.doi.org/10.1063/1.4880355]

In order to improve the performance of organic semiconductor devices such as organic light-emitting diodes (OLEDs), organic field-effect transistors (OFETs), and organic photovoltaic cells (OPVCs), a thorough understanding of charge transport properties of organic semiconductors is crucial. In the amorphous organic-semiconductor thin films in these devices, the disorder plays an important role. Stationary charge transport in disordered organic semiconductors has been studied to great depth, both experimentally and theoretically. Energetic disorder gives rise to a strong temperature, $T$, electric field, $F$, and charge-carrier density, $n$, dependence of the charge-carrier mobility, $\mu$, which is determined by the shape of the density of states (DOS). ${ }^{1-5}$ Nonstationary transport in organic devices, for which the stationary mobility function cannot be used, has not yet been studied to equal depth. An important question, addressed, in this Letter, is whether our understanding of nonstationary charge transport in these semiconductors can be reconciled with that of stationary transport.

Recently, we measured and simulated dark-injection (DI) current transients of hole-only devices of a polyfluorenebased light-emitting polymer (LEP). ${ }^{6}$ In DI experiments, a device that is kept in the dark is brought into a state of nonstationary charge transport by a sudden step in the applied voltage. The simulations were performed by solving a timedependent three-dimensional master equation (3D ME) for the occupational probabilities of sites representing localized states in the LEP. The temperature- and thickness-dependent stationary current density-voltage, $J-V$, characteristics of these devices had been analyzed before and a very good description was obtained within the extended Gaussian disorder model (EGDM) for the mobility function, ${ }^{4}$ using a standard deviation $\sigma=0.13 \mathrm{eV}$ of the Gaussian density of states. ${ }^{7-9}$ However, a proper modeling of the DI transients could only be obtained with a reduced value $\sigma=0.08 \mathrm{eV}$. This has brought up the important question whether our understanding of charge transport in disordered organic semiconductors is really complete.

Another important technique to study non-stationary transport in organic devices is impedance spectroscopy. In this technique, a dc bias $V$ is applied over a device and on top of that a small ac component $\Delta V(t)=\Delta V \sin (2 \pi \mathrm{i} f t)$. The impedance $Z=Z^{\prime}+\mathrm{i} Z^{\prime \prime}$ is defined as the zero-amplitude limiting value of the ratio of $\Delta V(t)$ and the response $\Delta I \exp [2 \pi \mathrm{i}(f+\phi) t]$ in the current, with $\phi$ is a phase difference. In particular, we will be interested in the capacitancevoltage, $C-V$, characteristics, with the capacitance given by $C=-Z^{\prime \prime} / 2 \pi f|Z|^{2}$. Impedance spectroscopy can be fruitfully used to distinguish different trapping regimes in singlecarrier organic-semiconductor devices ${ }^{10}$ and to determine doping densities and built-in voltages in organic solar cells. ${ }^{11}$

Modeling of the $C-V$ characteristics with the static mobility function neglects the effects of carrier relaxation in the DOS. Recently, we developed a multiple-trapping model for this carrier relaxation that is based on the existence of a conduction-level energy $E_{\mathrm{c}}{ }^{12}$ The idea is that the fraction of carriers around this energy in the DOS determines the conduction. Extra carriers induced by the fluctuating voltage slowly relax from this energy towards their equilibrium distribution. It was found that with a value $E_{\mathrm{c}}=-0.75 \sigma$ and the EGDM mobility function for the fraction of carriers at the conduction-level energy a fair description of the $C-V$ characteristics at different frequencies of the polyfluorene-based hole-only devices could be obtained with $\sigma=0.13 \mathrm{eV} .^{12}$ However, $E_{\mathrm{c}}$ was essentially treated as a fit parameter in the modeling, so that hard conclusions about the comparison between stationary and nonstationary charge transport could not be drawn.

In this Letter, we follow the same methodology as in our modeling of DI transients ${ }^{6}$ and simulate the impedance 
spectroscopy experiments by explicitly solving a timedependent 3D ME for a lattice of transport sites representing a complete device. The great advantage of this methodology is that it does not suffer from simplifications inherent to onedimensional (1D) modeling and provides information at the microscopic level. Like in the modeling of DI transients, this makes a direct comparison between stationary and nonstationary charge transport possible.

The time-dependent 3D ME for the occupational probabilities $p_{i}$ of sites $i$ in the device is given by

$$
\frac{d p_{i}}{d t}=\sum_{j \neq i}\left[W_{j i} p_{j}\left(1-p_{i}\right)-W_{i j} p_{i}\left(1-p_{j}\right)\right] \equiv g_{i}(\mathbf{p}),
$$

where $W_{i j}$ is the hopping rate from site $i$ to $j$ and $\mathbf{p}$ is the vector of all $p_{i}$ 's. We follow the same procedure as in Ref. 13 in finding the steady-state solution $\mathbf{p}_{0}$ for $d p_{i} / d t=0$ at the applied static voltage $V$. Sheets of sites representing the electrodes are introduced at either side of the simulation box in the same way as in Ref. 13. Since a small-amplitude ac-signal with the frequency $f$ induces a minor change $\Delta \mathbf{p}$, we write $\mathbf{p}(t) \approx \mathbf{p}_{0}+\exp (2 \pi \mathrm{i} f t) \Delta \mathbf{p}$ and $\mathbf{g}(\mathbf{p}) \approx \mathbf{g}\left(\mathbf{p}_{0}\right)$ $+\exp (2 \pi \mathrm{i} f t)[\Delta V \partial \mathbf{g} / \partial V+\mathbf{J} \Delta \mathbf{p}]$, with the matrix elements of the Jacobian $\mathbf{J}$ given by $J_{i j}=\partial g_{i} /\left.\partial p_{j}\right|_{\mathbf{p}_{0}}$. Substituting these expressions in Eq. (1) and linearizing leads to the equation

$$
(2 \pi i f \mathbf{I}+\mathbf{J}) \Delta \mathbf{p}=-\Delta V \frac{\partial \mathbf{g}}{\partial V},
$$

with $\mathbf{I}$ is the identity matrix. Equation (2) can be solved for $\Delta \mathbf{p}$ and from this the current $\Delta I$ and the capacitance $C$ are straightforwardly obtained. Like in Ref. 6, we draw the site energies from a Gaussian DOS with standard deviation $\sigma$ and assume Miller-Abrahams hopping rates ${ }^{14}$

$$
W_{i j}=w_{0} \times\left\{\begin{array}{lll}
\exp \left[-\Delta E_{i j} / k_{\mathrm{B}} T\right] & \text { if } & \Delta E_{i j}>0 \\
1 & \text { if } & \Delta E_{i j} \leq 0
\end{array}\right.
$$

where $\Delta E_{i j}$ is the energy difference between sites $j$ and $i$, which includes the energetic disorder as well as the electrostatic potential due to a perpendicular layer-averaged electric field in the device. ${ }^{13} \mathrm{We}$ assume that the hopping takes place between nearest neighbors. We use a simple cubic (SC) as well as a face-centered cubic (FCC) lattice in our simulations. A factor related to wave function overlap that decays exponentially with distance, but is the same for each pair of nearest neighbors in a regular lattice, has been included in the prefactor $w_{0}$.

We performed simulations for the same two devices as studied in Ref. 12. They have the structure glass, indium tin oxide $(100 \mathrm{~nm})$, poly(3,4-ethylene-dioxythiophene):poly (styrenesulphonic acid) (PEDOT:PSS) (100 nm), LEP, Pd $(100 \mathrm{~nm})$. The LEP consists of polyfluorene with copolymerized triarylamine units $(7.5 \mathrm{~mol}$. \%) for hole transport; see Fig. 1(a). The LEP-layer thicknesses are $L=97$ and $121 \mathrm{~nm}$ for the two devices and their areas are $A=9 \times 10^{-6} \mathrm{~m}^{2}$. No injection barrier is taken at the anode (PEDOT:PSS) and injection barriers of 1.65 and $1.90 \mathrm{eV}$ are taken at the cathode (Pd) for the $L=97$ and $L=121 \mathrm{~nm}$ device, respectively, according to EGDM modeling studies of the $J$ - $V$ characteristics of these devices. ${ }^{7,15}$ These modeling studies gave best fits

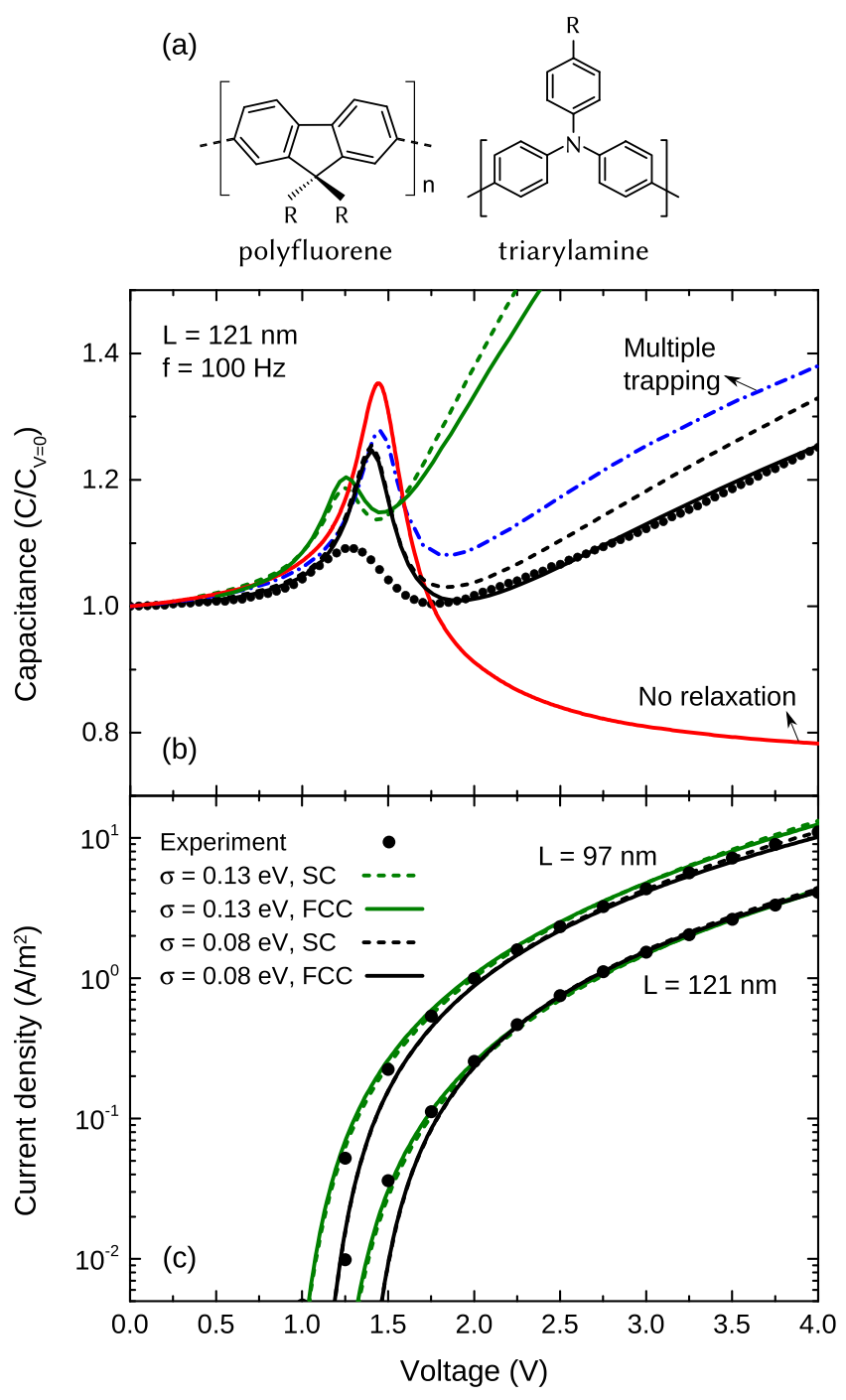

FIG. 1. (a) Schematic structure of the polyfluorene-triarylamine copolymer, (b) capacitance $C$, normalized to its value at $V=0$, as a function of $V$ at a frequency $f=100 \mathrm{~Hz}$ and temperature $T=295 \mathrm{~K}$ for a hole-only device with a layer with thickness $L=121 \mathrm{~nm}$. Dots: measurements. ${ }^{12}$ Red line: 1D DD calculation with an EGDM mobility function for $\sigma=0.13 \mathrm{eV}$, neglecting relaxation. Dashed-dotted blue line: multiple-trapping result. ${ }^{12}$ (c) Current density-voltage, $J$ - $V$, characteristics of the $L=121$ and $L=97 \mathrm{~nm}$ device at $T=295$ K. Dots: measurements. ${ }^{6}$ Green lines in both (b) and (c): 3D ME simulations for $\sigma=0.13 \mathrm{eV}$. Black lines: 3D ME simulations for $\sigma=0.08$. Dashed (full) green and black lines: SC (FCC) lattice.

for the $J-V$ characteristics with $\sigma=0.13 \mathrm{eV}$. The relative dielectric constant of the LEP is $\epsilon_{\mathrm{r}}=3.2$. $^{7}$ The simulation boxes have dimensions $L \times L_{y} \times L_{z}$, with $L_{y}=L_{z}=50 \mathrm{a}$ and periodic boundary conditions in the $y$ - and $z$-direction, yielding a sufficient lateral averaging.

Figure 1(b) gives an overview of our main results for the $C-V$ characteristics of the $L=121 \mathrm{~nm}$ device at a frequency $f=100 \mathrm{~Hz}$ and room temperature $(295 \mathrm{~K})$. We distinguish four regimes.

(1) At low voltage, all curves converge to the geometrical capacitance, because almost no carriers are present in the device.

(2) With increasing voltage, a sheet of holes builds up by diffusion at the anode, but these cannot yet move to the cathode because the electric field is still directed from cathode to anode. As a result, the capacitance rises. 
(3) When approaching the built-in voltage $V_{\mathrm{bi}}(1.9 \mathrm{~V}$ in this case), these holes start to move to the cathode, leading to a decrease of the capacitance. The result is a peak in the $C$ - $V$ curve before $V_{\mathrm{bi}}$ is reached. ${ }^{16}$

(4) Beyond $V_{\mathrm{bi}}$ a regime starts where the experimental $C-V$ curve rises again and where the different modeling results predict very different behavior. It is this regime we want to focus on.

The red curve in Fig. 1(b) is a modeling result in which a time-dependent one-dimensional drift-diffusion (1D DD) equation is solved with the EGDM mobility function for $\sigma=0.13 \mathrm{eV} .{ }^{12}$ This model assumes that the mobility at any moment in time is given by the instantaneous carrier density and electric field through this function, and therefore does not account for carrier relaxation. At high voltages, the curve deviates strongly from experiment, which should be attributed to the neglect of relaxation. The dashed-dotted blue curve is the result of the multiple-trapping model for relaxation, assuming a conduction-level energy $E_{\mathrm{c}}=-0.75 \sigma$. The agreement with experiment is in this case fair, not only at the frequency $f=100 \mathrm{~Hz}$ but also at the other frequencies investigated in Ref. 12 (not shown here).

However, the $C-V$ curves obtained from the 3D ME simulations for $\sigma=0.13 \mathrm{eV}$ do not agree at all with experiment. The dashed green curve gives the result obtained for a SC lattice, with a lattice constant $a=1.19 \mathrm{~nm}$. The latter value was obtained from the value $N_{t}=6 \times 10^{26} \mathrm{~m}^{-3}=1 / a^{3}$ for the density of transport sites found in the modeling studies of Ref. 7, a value that is compatible with the known density of $1.8 \times 10^{26} \mathrm{~m}^{-3}$ of triarylamine units in the used copolymer. The effects of carrier relaxation are clearly too strong in this case.

In Fig. 1(c), we compare the corresponding result for the $J-V$ curve with experiment, leading to an excellent agreement. The value of the hopping prefactor $w_{0}$ found from this comparison is given in Table I. We conclude that there is a strong discrepancy between the description of the stationary charge transport reflected by the $J-V$ curve and the nonstationary charge transport reflected by the $C-V$ curve.

One may argue that carrier relaxation could depend on the type of lattice and, in particular, on the number of nearest neighbors. To investigate this, we also performed simulations for an FCC lattice with a lattice constant of $a=1.88 \mathrm{~nm}$, corresponding to the same site density as in the SC lattice, but with 12 instead of 6 nearest neighbors. It was found from numerical calculations that the critical energy for percolation within the EGDM is significantly lower for an FCC than for a SC lattice, due to the larger number of nearest neighbors. ${ }^{17}$ One may wonder if this could mean that also the conduction level energy $E_{\mathrm{c}}$ is lower, leading to weaker

TABLE I. Values for the hopping prefactor $w_{0}$ in Eq. (3) obtained from fits to the measured $J$ - $V$ curves of Fig. 1(c).

\begin{tabular}{lcc}
\hline \hline & \multicolumn{2}{c}{$w_{0}\left(\mathrm{~s}^{-1}\right)$} \\
\cline { 2 - 3 }$\sigma(\mathrm{eV})$ & $\mathrm{SC}$ & $\mathrm{FCC}$ \\
\hline 0.13 & $2.7 \times 10^{10}$ & $3.2 \times 10^{9}$ \\
0.08 & $9.1 \times 10^{7}$ & $2.2 \times 10^{7}$ \\
\hline \hline
\end{tabular}

relaxation effects. Figure 1(c) shows that again excellent agreement is obtained with the experimental $J-V$ curve, with an adapted value of $w_{0}$ given in Table I. The full green curve in Fig. 1(b) is the $C-V$ result for this case. This curve lies only slightly below the SC curve from which we conclude that the type of lattice only has a minor influence on relaxation.

In the modeling of DI transients in Ref. 6, it was also found that unsatisfactory agreement with experiment was found when taking the same value for $\sigma$ in the DI modeling as in the modeling of the $J-V$ curves. Reducing the value of $\sigma$ to $0.08 \mathrm{eV}$, however, led to a very good agreement. We tested whether taking this reduced value of $\sigma$ leads also to an improvement in the modeling of the $C$ - $V$ curves. To this end, we first refitted the $J-V$ curves with $\sigma=0.08 \mathrm{eV}$. Figure 1(c) shows that very satisfactory fits are obtained at higher voltages, both for the SC and FCC lattice. Only at low voltages $(<2 \mathrm{~V})$, the fits are inferior to those with $\sigma=0.13 \mathrm{eV}$. The results obtained for $w_{0}$ are again given in Table I. Figure 1(b) shows that indeed the $C-V$ curves are now in very good agreement with experiment, with the FCC curve even falling on top of the experimental curve for voltages $>2 \mathrm{~V}$. The only clear disagreement is in the peak, which is more pronounced in the modeling than in the experiment. In Ref. 12, it was suggested that lateral variations in $V_{\mathrm{bi}}$ will lead to widening of the experimental peak, which may partially explain the disagreement.

Figure 2 shows comparisons between experimental and simulated $C$ - $V$ curves for $\sigma=0.08 \mathrm{eV}$ and for both SC and FCC lattices, at frequencies of 100,250 , and $1000 \mathrm{~Hz}$, for
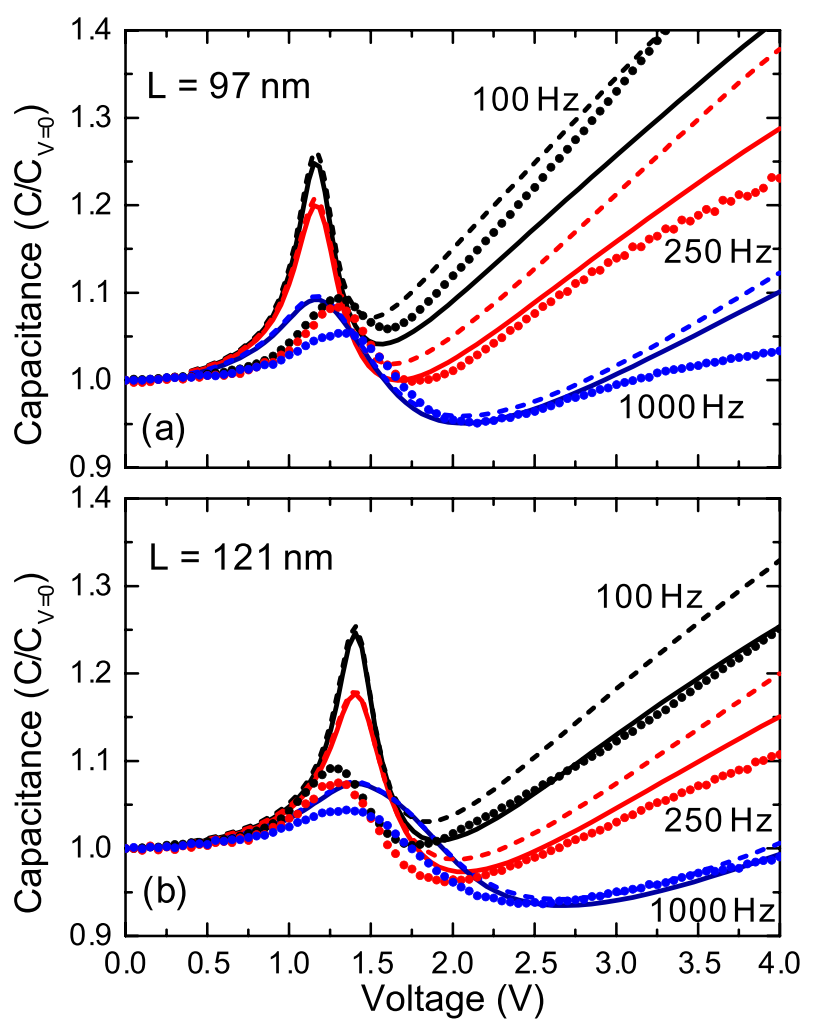

FIG. 2. Capacitance $C$, normalized to its value at $V=0$, as a function of $V$ at different frequencies for (a) the $L=97 \mathrm{~nm}$ and (b) the $L=121$ device. Dots: measurements. ${ }^{12}$ Full (dashed) lines: simulation results for $\sigma=0.08 \mathrm{eV}$ and a SC (FCC) lattice. 
the $L=97 \mathrm{~nm}$ (Fig. 2(a)) and $L=121 \mathrm{~nm}$ (Fig. 2(b)) device. The conclusion is that the overall agreement is very satisfactory. At the highest frequency of $1000 \mathrm{~Hz}$, relaxation effects are the least significant. This can be understood from Eq. (2), in which at high frequency $f$ the first, "direct," term on the left-hand side becomes dominant with respect to the second, "indirect," term, which accounts for the relaxation effects.

To exclude the possibility that the obtained agreement with the experimental $C-V$ curves using $\sigma=0.08 \mathrm{eV}$ is fortuitous, we considered other factors that could influence carrier relaxation. Specifically, we looked at variablerange instead of nearest-neighbor hopping and Marcus ${ }^{18}$ instead of Miller-Abrahams hopping rates. In the case of variable-range hopping $(\mathrm{VRH})$, a prefactor $\exp \left(-2 \alpha R_{i j}\right)$ should be included in Eq. (3), where $R_{i j}$ is the distance between sites $i$ and $j$, and $\alpha$ is the inverse wave function decay length. We have no information about $\alpha$ for our case, but taking $\alpha=4 / a$ leads to considerable rates for further than nearest-neighbor hops. For this value and taking hopping up to the fourth nearest-neighbor into account on an SC lattice, we found that some effect of VRH is visible on the $C-V$ curves at high voltage, but the effect is significantly smaller than the difference between the results for $\sigma=0.13$ and $0.08 \mathrm{eV}$ in Fig. 1(b). Regarding Marcus hopping rates it has been found that the mobility function and hence the stationary charge transport is not significantly different than for Miller-Abrahams hopping rates, ${ }^{17}$ but it is not a priori clear that the same holds for carrier relaxation. Information about the reorganization energy $E_{\mathrm{r}}$ in the Marcus hopping rate is lacking in our case. Our master-equation solver allowed the study of Marcus hopping on an SC lattice for reorganization energies down to $E_{\mathrm{r}} \approx 3 \sigma$. For all considered cases, we found that the difference with Miller-Abrahams hopping rates is small.

The puzzling question remains why there is such a discrepancy between the description of stationary and nonstationary charge transport. A possible explanation was suggested in Ref. 6: maybe in the stationary transport the low-energy tail of the DOS is important, represented by a relatively large $\sigma$, while in nonstationary transport relaxing carriers probe a larger part of the DOS, represented by a smaller $\sigma$. In other words, the shape of the DOS could be more complicated than a single Gaussian. This explanation seems to be corroborated by the $J-V$ curves in Fig. 1(c). At low voltage, $\sigma=0.13 \mathrm{eV}$ gives a better description, while at higher voltage $\sigma=0.08 \mathrm{eV}$ gives an excellent and even a slightly better description. A value of $\sigma=0.13 \mathrm{eV}$ at low voltage could also explain the lower peak in the $C$ - $V$ curve as compared to the simulations with $\sigma=0.08 \mathrm{eV}$ in Figs. 1(b) and 2. We remark here that the positions of the peak could be improved by adapting the built-in voltages $V_{\mathrm{bi}}$ used in the calculations. We recall that these voltages were obtained from an EGDM fit of the $J-V$ characteristics with $\sigma=0.13 \mathrm{eV}$, but the obtained values are probably not optimal for $\sigma=0.08 \mathrm{eV}$.

It was remarked in Ref. 6 that a single-Gaussian DOS with $\sigma=0.08 \mathrm{eV}$ will lead to a temperature dependence in the mobility in the limit of vanishing carrier density that is too weak as compared to experiment. In the scenario of a more complicated DOS sketched here, however, it would be the value $\sigma=0.13 \mathrm{eV}$ that determines the temperature dependence in this limit, which is known to lead to a good agreement with experiment. ${ }^{7}$ We suggest that other types of experiments are performed on our system that can more directly probe the shape of the DOS. Specifically, we think of stimulated current (TSC) measurements, which have originally been used to extract information about trap levels, ${ }^{19-21}$ but could also be employed more generally to study DOS tails.

This work was supported by the Dutch nanotechnology program NanoNextNL (M.M.) and the Dutch Polymer Institute (DPI), Project No. 680 (J.C.). We thank MSc Harm van Eersel for a critical reading of the manuscript.

${ }^{1}$ H. Bässler, Phys. Status Solidi B 175, 15 (1993).

${ }^{2}$ M. C. J. M. Vissenberg and M. Matters, Phys. Rev. B 57, 12964 (1998).

${ }^{3}$ C. Tanase, E. J. Meijer, P. W. M. Blom, and D. M. de Leeuw, Phys. Rev. Lett. 91, 216601 (2003).

${ }^{4}$ W. F. Pasveer, J. Cottaar, C. Tanase, R. Coehoorn, P. A. Bobbert, P. W. M. Blom, D. M. de Leeuw, and M. A. J. Michels, Phys. Rev. Lett. 94, 206601 (2005).

${ }^{5}$ V. Coropceanu, J. Cornil, D. A. da Silva Filho, Y. Olivier, R. Silbey, and J.-L. Brédas, Chem. Rev. 107, 926 (2007).

${ }^{6}$ M. Mesta, C. Schaefer, J. de Groot, J. Cottaar, R. Coehoorn, and P. A. Bobbert, Phys. Rev. B 88, 174204 (2013).

${ }^{7}$ S. L. M. van Mensfoort, S. I. E. Vulto, R. A. J. Janssen, and R. Coehoorn, Phys. Rev. B 78, 085208 (2008).

${ }^{8}$ R. J. de Vries, S. L. M. van Mensfoort, V. Shabro, S. I. E. Vulto, R. A. J. Janssen, and R. Coehoorn, Appl. Phys. Lett. 94, 163307 (2009).

${ }^{9}$ R. J. de Vries, A. Badinski, R. A. J. Janssen, and R. Coehoorn, J. Appl. Phys. 113, 114505 (2013).

${ }^{10}$ E. Knapp and B. Ruhstaller, Appl. Phys. Lett. 99, 093304 (2011).

${ }^{11}$ R. C. I. MacKenzie, C. G. Shuttle, G. F. Dibb, N. Treat, E. von Hauff, M. J. Robb, C. J. Hawker, M. L. Chabinyc, and J. Nelson, J. Phys. Chem. C 117, 12407 (2013).

${ }^{12}$ W. C. Germs, J. J. M. van der Holst, S. L. M. van Mensfoort, P. A. Bobbert, and R. Coehoorn, Phys. Rev. B 84, 165210 (2011).

${ }^{13}$ J. J. M. van der Holst, M. A. Uijttewaal, B. Ramachandhran, R. Coehoorn, P. A. Bobbert, G. A. de Wijs, and R. A. de Groot, Phys. Rev. B 79, 085203 (2009).

${ }^{14}$ A. Miller and E. Abrahams, Phys. Rev. 120, 745 (1960).

${ }^{15}$ R. J. de Vries, S. L. M. van Mensfoort, R. A. J. Janssen, and R. Coehoorn, Phys. Rev. B 81, 125203 (2010).

${ }^{16}$ S. L. M. van Mensfoort and R. Coehoorn, Phys. Rev. Lett. 100, 086802 (2008).

${ }^{17}$ J. Cottaar, L. J. A. Koster, R. Coehoorn, and P. A. Bobbert, Phys. Rev. Lett. 107, 136601 (2011).

${ }^{18}$ R. A. Marcus, Rev. Mod. Phys. 65, 599 (1993).

${ }^{19}$ A. G. Werner, J. Blochwitz, M. Pfeiffer, and K. Leo, J. Appl. Phys. 90, 123 (2001).

${ }^{20}$ W. Weise, T. Keith, N. von Malm, and H. von Seggern, Phys. Rev. B 72, 045202 (2005).

${ }^{21}$ P. Yu, A. Migan-Dubois, J. Alvarez, A. Darga, V. Vissac, D. Mencaraglia, Y. Zhou, and M. Krueger, J. Non-Cryst. Solids 358, 2537 (2012). 